Please do not remove this page

RMIT

UNIVERSITY

\title{
Observation of nonlinear optical Tamm states in truncated photonic lattices
}

Rosberg, Christian; Neshev, Dragomir; Krolikowski, Wieslaw; Mitchell, Arnan; Vicencio, Rodrigo; Molina, Mario; Kivshar, Yuri

https://researchrepository.rmit.edu.au/esploro/outputs/9921859934801341/filesAndLinks?institution=61RMIT_INST\&index=null

Rosberg, C., Neshev, D., Krolikowski, W., Mitchell, A., Vicencio, R., Molina, M., \& Kivshar, Y. (2006).

Observation of nonlinear optical Tamm states in truncated photonic lattices. Proceedings of 2006 International Conference on Nanoscience and Nanotechnology (ICONN 2006).

https://doi.org/10.1109/ICONN.2006.340653

Published Version: https://doi.org/10.1109/ICONN.2006.340653

Repository homepage: https://researchrepository.rmit.edu.au

(c) 2006 IEEE. Personal use of this material is permitted. However, permission to reprint/republish this material for advertising or promotional purposes or for creating new collective works for resale or redistribution to servers or lists, or to reuse any copyrighted component of this work in other works must be obtained from the IEEE.

Downloaded On 2023/04/26 14:47:53 +1000 


\title{
Observation of nonlinear optical Tamm states in truncated photonic lattices
}

\author{
Christian R. Rosberg*, Dragomir N. Neshev*, Wieslaw Krolikowski ${ }^{\dagger}$, Arnan Mitchell ${ }^{\ddagger}$, \\ Rodrigo A. Vicencio ${ }^{\S}$, Mario I. Molina*, and Yuri S. Kivshar* \\ * Nonlinear Physics Centre and ${ }^{\dagger}$ Laser Physics Centre, \\ Centre for Ultrahigh-bandwidth Devices for Optical Systems (CUDOS), \\ Research School of Physical Sciences and Engineering, \\ Australian National University, Canberra, Australia \\ Email: crr124@rsphysse.anu.edu.au \\ ${ }^{\ddagger}$ School of Electrical and Computer Systems Engineering, \\ RMIT University, Melbourne, Australia \\ ${ }^{\S}$ Max-Planck-Institut für Physik komplexer Systeme, Dresden, Germany \\ -Departamento de Física, Facultad de Ciencias, \\ Universidad de Chile, Santiago, Chile
}

\begin{abstract}
We report on the first observation of surface gap solitons, a nonlinear optical analog of localized surface Tamm states in periodic electronic potentials. We demonstrate strong beam self-trapping in the form of staggered surface modes at the interface between uniform and periodic dielectric media with defocusing nonlinear response.
\end{abstract}

Keywords-nonlinear optics; optical solitons; periodic media; nonlinear waveguides; surface waves; Tamm states

\section{INTRODUCTION}

The existence of localized surface waves was first put forward in 1932 by Tamm [1]. He considered a truncated electronic potential which under certain conditions was found to support localized states at the surface, now commonly referred to as "Tamm states". Because of the difficulties in observing electronic surface waves in natural materials such as crystals, successful efforts have been made to experimentally demonstrate their existence in nano-engineered periodic layered structures or superlattices [2]. In optics, linear surface waves similar to electronic Tamm states have been observed in periodic GaAs-AlGaAs structures [3], exploiting the wellknown analogy between wave phenomena in optical and electronic periodic systems.

In this work, we demonstrate experimentally the existence of self-localized optical surface waves at the edge of a defocusing waveguide array acting as a truncated (semiinfinite) nonlinear photonic lattice. We observe, for the first time to our knowledge, the formation of nonlinear optical Tamm states or surface gap solitons, recently predicted in theory [4]. Surface gap solitons exhibit a nontrivial staggered phase structure, and a propagation constant lying within the first photonic transmission bandgap of the periodic structure [5]. The defocusing nonlinear response of the material enables the formation of surface localized and staggered modes, and thus allows for a full nonlinear analogy with the electronic Tamm states.
Nonlinear surface waves with trivial phase structure, localized due to the mechanism of total internal reflection, were recently observed at the edge of self-focusing waveguide arrays [6]. They differ substantially from the staggered surface gap solitons, for which the localization mechanism consists of total internal reflection from the homogeneous region

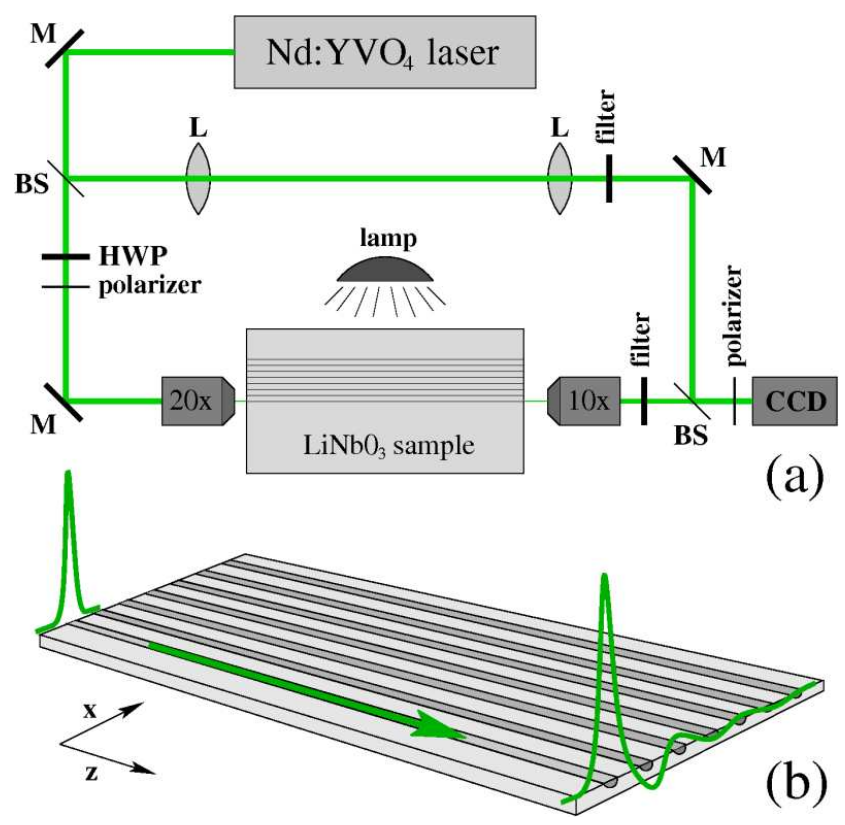

Figure 1. (a) Experimental setup. An extraordinarily polarized laser beam is focused onto the edge waveguide of a semi-infinite waveguide array by a microscope objective. The output is imaged onto a CCD camera. A plane reference wave is used to detect the phase structure of the output beam. M, BS, L, and HWP refer to mirror, beam splitter, lens and half wave plate, respectively. (b) Schematic illustration of the excitation of nonlinear optical Tamm states (surface gap solitons) by a narrow Gaussian input beam. 
combined with Bragg reflection from the semi-infinite periodic structure.

\section{EXPERIMENT}

The experiment is performed in an array of closely spaced single-mode waveguides fabricated by Titanium indiffusion in a mono-crystal lithium niobate wafer. In the fabrication process, $100 \AA$ of $\mathrm{Ti}$ was deposited on an X-cut LiNbO3 wafer using electron beam evaporation. The $\mathrm{Ti}$ layer was then photolithographically patterned and etched in a buffered hydrofluoric acid solution. The diffusion was conducted at $1050{ }^{\circ} \mathrm{C}$ for 3 hours in a wet oxygen environment. The resulting refractive index contrast is $\Delta \mathrm{n}=3 \cdot 10^{-4}$, the waveguide spacing $d=9.0 \mu \mathrm{m}$, the number of waveguides 100 , and the propagation length $50 \mathrm{~mm}$. The $\mathrm{LiNbO}_{3}$ sample exhibits a strong photovoltaic effect which leads to a saturable selfdefocusing nonlinearity.

Figure 1(a) shows a schematic drawing of the experimental setup. At the input face of the waveguide sample, a circular shaped and extraordinarily polarized probe beam from a $\mathrm{cw}$ $\mathrm{Nd}: \mathrm{YVO}_{4}$ laser at wavelength $\lambda=532 \mathrm{~nm}$ is focused by a microscope objective $(\times 20)$ to a full width at half-maximum (FWHM) of $3 \mu \mathrm{m}$, and injected into the waveguide at the edge of the array [Fig. 1]. It was recently shown in Ref. [7] that, provided the refractive index difference exceeds a certain value, such single-site excitation constitutes a simple and effective method for exciting nonlinear localized staggered modes in defocusing periodic structures, thus avoiding a specific phase preparation of the input beam. In this case the periodic structure appears equivalent to a discrete system and can be described by a discrete nonlinear model. The propagated wave packet at the sample output is imaged onto a CCD camera to capture the intensity distribution. The waveguide array is externally illuminated by a white-light source in order to control the nonlinear response time. A plane reference wave is used to map the phase structure of the output beam. Figure 1(b) illustrates part of the semi-infinite photonic lattice geometry, as well as the formation of localized nonlinear optical Tamm states or surface gap solitons, by excitation with a high power narrow Gaussian input beam. $x$ and $z$ are the transverse and longitudinal (propagation) coordinates, respectively.

At low laser power $(0.1 \mu \mathrm{W})$ the probe beam experiences discrete diffraction and spreads out in the horizontal plane, due to strong coupling between neighboring waveguides and repulsion from the surface. Figure 2(a) depicts the experimental output image and the corresponding transverse intensity profile in this situation. After linear propagation through the array the beam profile spans more than ten waveguides, and the maximum is centered approximately 42 lattice sites away from the input excitation point (the edge of the array). Figure 2(b) shows the corresponding numerically calculated intensity distribution inside the sample, obtained by using a simple analytical formula derived from a discrete model [8]. The excellent agreement between the experimental observation and the theoretical prediction yields a value of $0.46 \mathrm{~mm}^{-1}$ for the intersite coupling coefficient of the waveguide array, implying a total longitudinal propagation of 23 coupling lengths.
Increasing the laser power leads to beam self-action in the nonlinear regime. The slow response of the photovoltaic nonlinearity allows us to monitor the dynamics of the soliton formation, providing additional information about the localization process. Figs. 2(c-e) show the output beam intensity profile at times 920, 1050, and $1550 \mathrm{~s}$, respectively, after the beam power is increased to $0.5 \mathrm{~mW}$. The wave packet is seen to first contract considerably and move towards the edge of the array [Fig. 2(c)]. Then, as shown in Fig. 2(d), partial self-trapping in the surface waveguide becomes apparent. At this stage the beam still possesses a long tail of intensity lobes extending into the periodic medium. A series of zero intensity points between these lobes indicates the selfinduced dynamic formation of a staggered phase structure. Eventually, a strongly localized nonlinear Tamm state or surface gap soliton is formed [Fig. 2(e)]. The asymmetry of the photonic structure is reflected in the shape of the trapped beam which monotonically decays above the surface, but oscillates inside the periodic region. The damped oscillations resemble the shape of Bloch modes near the edge of the first transmission band of the periodic structure. At the interface between the array and the uniform region, the defocusing nonlinearity is manifested by the fact that it effectively decreases the contrast of the surface waveguide, causing the localized mode to broaden and penetrate substantially into the homogeneous region [Fig. 2(e)].
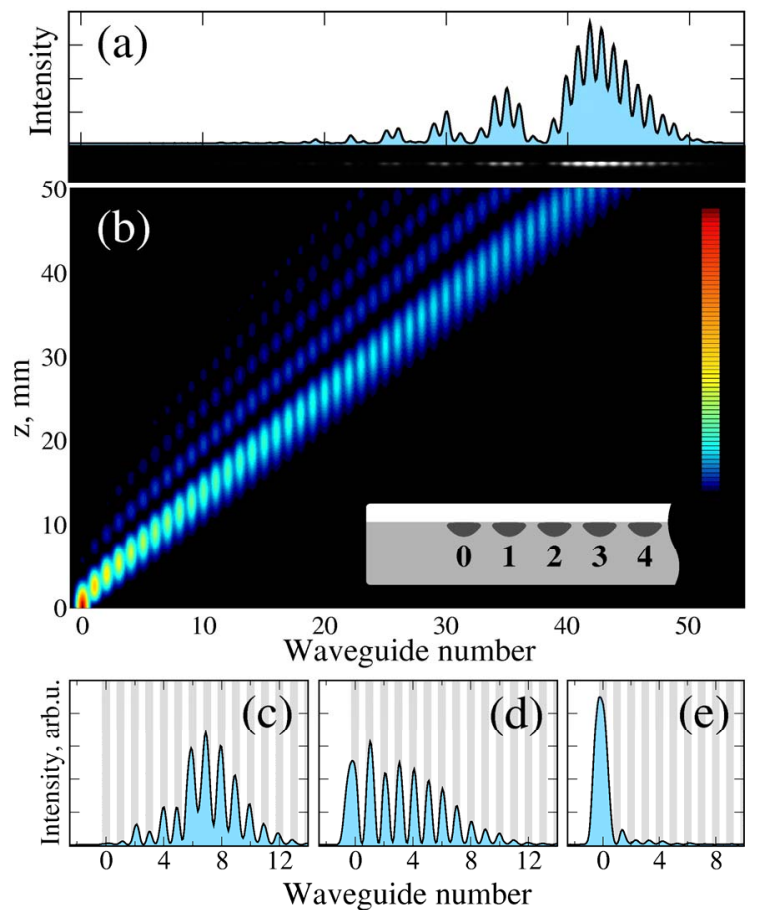

Figure 2. (a) Measured and (b) calculated linear diffraction of a narrow low power beam $(0.1 \mu \mathrm{W})$ when only the edge waveguide is excited. Inset in (b) illustrates the waveguide geometry. (c-e) Nonlinear Tamm state formation. Pictures show the output intensity distribution at times 920,1050, and $1550 \mathrm{~s}$, respectively, after beam power is increased to $0.5 \mathrm{~mW}$. Grey shading marks the waveguide positions. 


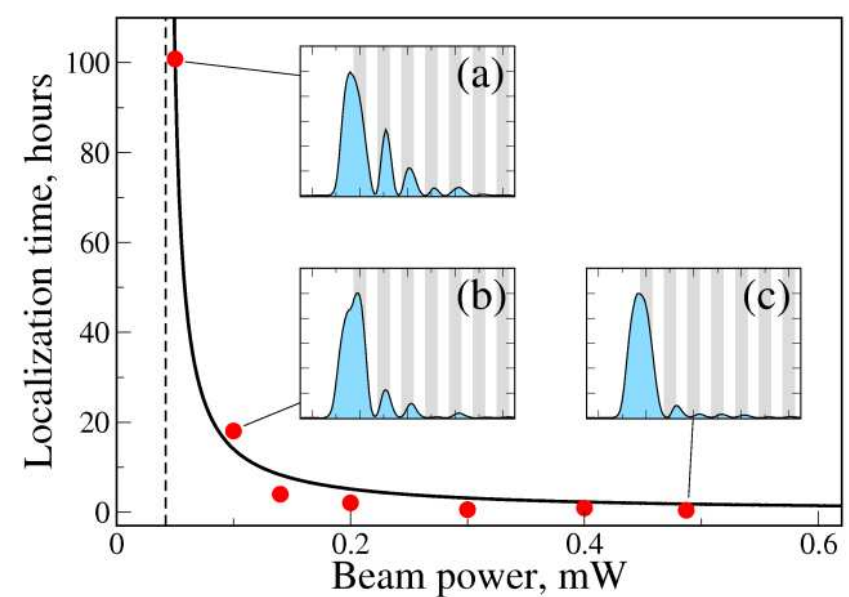

Figure 3. Measured surface localization time versus probe beam power. Solid curve: $\mathrm{A}+\mathrm{B} /\left(\mathrm{P}-\mathrm{P}_{\mathrm{th}}\right)$ fit to eksperimental data (dots). Vertical dashed line marks the threshold power $\left(\mathrm{P}_{\mathrm{th}}=0.042 \mathrm{~mW}\right)$. (a-c) Beam intensity profiles of decreasing width, corresponding to the indicated data points.

In order to study in detail the crossover between linear diffraction and nonlinear self-localization, we measure the formation time of the surface state as a function of the probe beam power. The results are summarized in Fig. 3. The formation time is seen to increase dramatically for decreasing input power until, below a certain critical power, no localized surface mode is formed. This so-called critical slowing down indicates the existence of a threshold power below which the nonlinear response is too weak to cause self-trapping. The value of the threshold power was estimated as $\mathrm{P}_{\mathrm{th}}=0.042 \mathrm{~mW}$ by modeling the dynamics of the soliton formation time, fitting the function $\mathrm{A}+\mathrm{B} /\left(\mathrm{P}-\mathrm{P}_{\text {th }}\right)$ (Fig. 3, solid curve) to the experimental data [Fig. 3, dots]. Figures 3(a-c) depict the beam intensity profiles corresponding to the indicated data points. The width of the localized mode decreases for increasing beam power, spanning about three lattice sites immediately above threshold [see Fig. 3(a)], and approximately a single lattice site at high power, as indicated in Fig. 3(c). The decrease of the beam width is caused by the fact that stronger beam self-action at higher power leads to a deeper surface defect, and hence to more pronounced beam localization.

The staggered phase structure of the part of the field extending into the periodic medium represents an essential and unique feature associated with nonlinear Tamm states. The alternating phase of the beam tail inside the waveguide array reflects the fact that the propagation constant of the selflocalized mode lies within the Bragg reflection bandgap. To verify the nontrivial phase structure in the experiment we interfere the output beam with a vertically inclined broad plane reference beam and image the obtained interferogram (Fig. 4).

Figure 4(a) depicts a three dimensional representation of the spatial beam intensity distribution of a broad surface gap soliton observed immediately above threshold power $(0.05$ $\mathrm{mW}$ ). Figure 4(b) shows the intensity plot of the associated interference pattern, where $x$ and $y$ are the horizontal and vertical coordinates, respectively [cf. Fig. 1(b)]. A half-period vertical shift of the interference fringes, corresponding to an exact $\pi$ phase jump in the horizontal beam direction, is clearly observed between each pair of lobes in the structure [Fig. 4(b)].

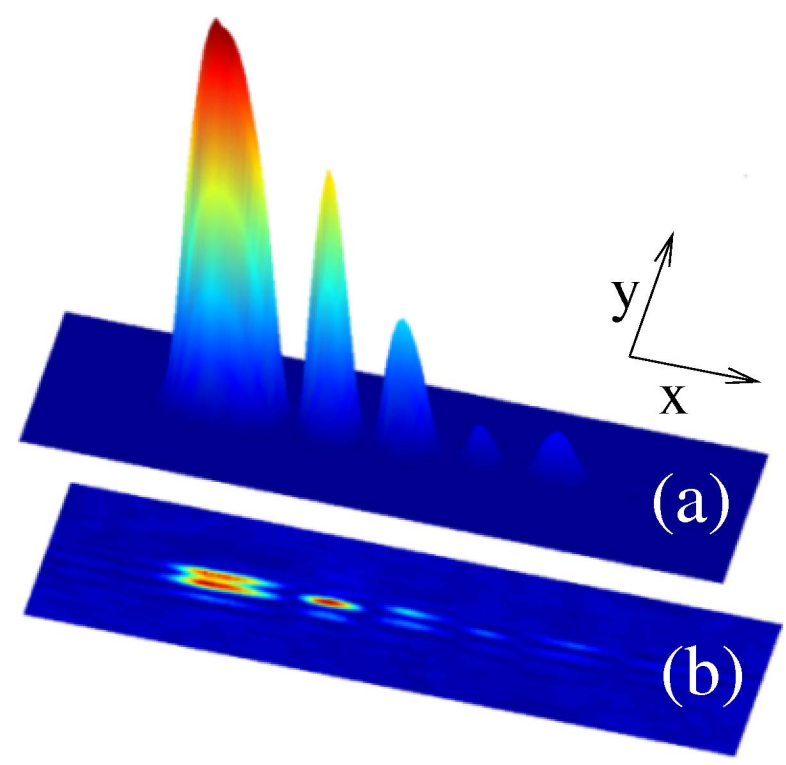

Figure 4. (a) Three dimensional representation of the observed nonlinear Tamm state (surface gap soliton) immediately above threshold power [corresponding to Fig. 3(a)]. $x$ and $y$ are the horizontal and vertical coordinates, respectively. (b) Plane wave interferogram demonstrating the staggered phase structure of the surface state.

\section{NONLINEAR DISCRETE MODEL}

In order to gain deeper insight into the physics of the staggered surface soliton formation in semi-infinite lattices with defocusing nonlinearity, we consider a system of coupledmode equations for the normalized mode amplitudes $E_{0}$ and $E_{n}$ $(n=1,2, \ldots)$. Assuming weak coupling between the neighboring waveguides the equations read

$$
\begin{aligned}
& i \frac{d E_{0}}{d z}+E_{1}+\eta\left(E_{0}\right) E_{0}=0 \\
& i \frac{d E_{n}}{d z}+\left(E_{n+1}+E_{n-1}\right)+\eta\left(E_{n}\right) E_{n}=0
\end{aligned}
$$

where the function $\eta(E)=\gamma /\left(1+|E|^{2}\right)$ accounts for the saturable character of the defocusing photovoltaic nonlinearity $(\gamma>0)$. Looking for stationary solutions in the form $E_{n}(z)=$ $\exp (i \beta z) E_{n}$, we obtain the linear spectrum of extended modes, $\beta$ $=2 \cos k,(0 \leq k \leq \pi)$. No localized surface modes exist in the linear regime, as this would require very large index contrast between the waveguides and the continuum. However, the presence of a defocusing nonlinearity in the model can give rise to self-localized states. To find them we solve numerically the corresponding stationary equations by a multi-dimensional Newton-Raphson scheme. Since we are interested in surface localized modes, we look for solutions with maxima near the surface which decay quickly away from the edge of the array, similar to the earlier studied cases of Kerr nonlinearity for the discrete [8] and continuous [4] models.

Figure 5(a) shows an example of a calculated surface mode profile (the discrete mode amplitudes have been multiplied by the continuous waveguide mode-field profiles). Using the results solely based on the discrete model provides reasonable 

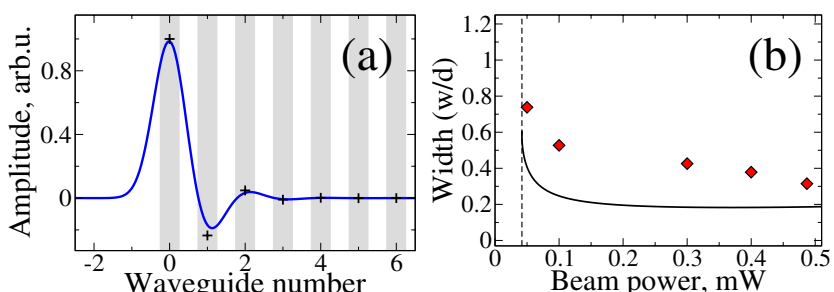

Figure 5. (a) Staggered surface mode calculated by use of a discrete nonlinear model $(\gamma=8)$, and expanded with continuous waveguide mode Gaussians. The discrete mode amplitudes are marked by (+) signs. (b) Normalized width of the surface state calculated numerically (solid curve) and measured experimentally (diamonds) as a function of the beam power. Vertical dashed line marks the threshold power $\left(\mathrm{P}_{\mathrm{th}}=0.042 \mathrm{~mW}\right)$.

agreement with the experimental data, as shown in Fig. 4(b) for the width of the localized surface state, calculated numerically (solid curve) and measured experimentally (diamonds) as a function of the beam power. Despite being approximate, the discrete model can be employed to reveal an important physical mechanism of the nonlinearity-induced surface mode stabilization. To this end we follow earlier studies $[9,10]$ and calculate the effective energy of the mode,

$$
H=-\sum_{n}\left\{\left(E_{n} E_{n+1}^{*}+E_{n}^{*} E_{n+1}\right)+\gamma \ln \left(1+\left|E_{n}\right|^{2}\right)\right\},
$$

as a function of its collective coordinate, defined as

$$
X=P^{-1} \sum_{n} n\left|E_{n}\right|^{2}
$$

where $P=\sum_{n}\left|E_{n}\right|^{2}$ is the mode power.

We apply a numerical constraint method and start from the solution centered at the site $n=0$ for given values of $\beta$ and $P$. Our goal is to obtain all intermediate solutions between the neighboring waveguides for the same power. First, we calculate the stationary mode centered at $n=0$ and obtain all $\left\{E_{n}\right\}$ and the power $P$. Then we fix the amplitude at the site $n=$ 1 to $E_{1}+\varepsilon$, and solve the Newton-Raphson equations for all remaining $E_{m}(m \neq 1)$ with the constraint that the power be kept at $P$, arriving at an intermediate state centered between $n=0$ and $n=1$. Finally we vary $\varepsilon$ and repeat the procedure until reaching the even configuration where $X=0.5$. The procedure is repeated for the solutions centered at $n=1,2,3$ which allows us to construct the effective potential plotted in Figs. 6(a,b).

Figures $6(a, b)$ show the effective potential of the surface modes in a semi-infinite array, $\mathrm{U}_{\text {eff }}(X) \equiv-H(X)$, calculated for two different power values. The extremal points of this curve, defined by the condition $d H / d X=0$, correspond to stationary localized solutions. In comparison with an infinite array, the truncation of the waveguide array introduces an effective repulsive surface potential, which is combined with the periodic potential of the array [Fig. 6(a)]. As a result, surface modes are possible neither in the linear regime nor in the continuous limit. As seen from Fig. 6(a), for low powers there exists no solution of the equation $d H / d X=0$ at the surface site $n=0$, and the surface repels the input beam, as observed in
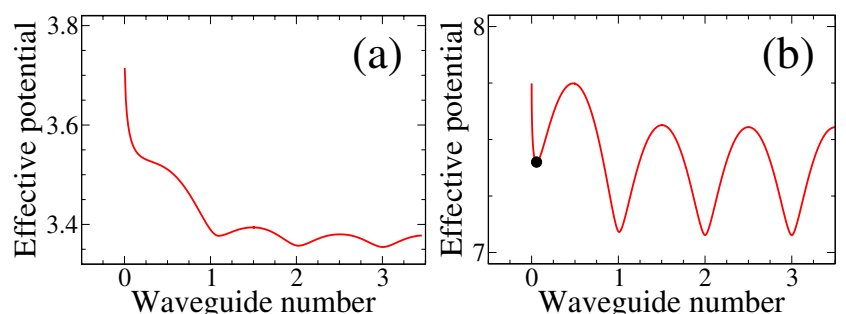

Figure 6. (a,b) Effective potential of the localized surface modes vs. the collective coordinate $X$ below and above the threshold power, respectively. Integer values of $X$ correspond to the waveguide numbers. Black dot in (b) refers to the stationary solution shown in Fig. 5(a).

experiment [see Fig. 2(a,b)]. However, when the input power exceeds the threshold value, discreteness overcomes the surface repulsive force and the localized state at $n=0$ in the form of a nonlinear Tamm state becomes possible [Fig. 6(b)].

\section{CONCLUSIONS}

We have demonstrated experimentally that a narrow optical beam can be trapped and stabilized near the surface of a semiinfinite periodic medium with self-defocusing nonlinearity in the form of staggered surface modes or surface gap solitons, providing the first evidence of a nonlinear analog of localized Tamm states in optics. While linear beams are repelled by the effective surface potential, the nonlinearity-induced defect locks the wave packet to the boundary of the periodic structure by tuning the propagation constant into the photonic bandgap, offering a unique possibility to dynamically control wave propagation at the interface between two different media. The effect demonstrated in this work has promising technical applications in areas such as all-optical switching and beam steering in integrated information processing systems.

The authors thank A.A. Sukhorukov for useful discussions. This work was supported by the Australian Research Council.

\section{REFERENCES}

[1] I. E. Tamm, "A possible kind of electron binding on crystal surfaces," Z. Phys. 76, 849 (1932).

[2] H. Ohno et al., "Observation of "Tamm states" in superlattices," Phys. Rev. Lett. 64, 2555-2558 (1990).

[3] Pochi Yeh, Amnon Yariv, and A. Y. Cho, "Optical surface waves in periodic layered media,” Appl. Phys. Lett. 32, 104-105 (1978).

[4] Y. V. Kartashov, V. A. Vysloukh, and L. Torner, "Surface gap solitons," Phys. Rev. Lett. 96, 073901 (2006).

[5] Yu. S. Kivshar and G. P. Agrawal, Optical Solitons: From Fibers to Photonic Crystals, Academic Press, San Diego, 2003.

[6] S. Suntsov et al., "Observation of discrete surface solitons," Phys. Rev. Lett. 96, 063901 (2006)

[7] M. Matuszewski et al., "Crossover from self-defocusing to discrete trapping in nonlinear waveguide arrays," Opt. Express 14, 254-259 (2006)

[8] K. G. Makris, S. Suntsov, D. N. Christodoulides, G. I. Stegeman, and A. Haché, "Discrete surface solitons," Opt. Lett. 30, 2466-2468 (2005).

[9] Yu. S. Kivshar, F. Zhang, and A. S. Kovalev, "Stable nonlinear heavymass impurity modes," Phys. Rev. B 55, 14265-14269 (1997).

[10] M. Molina, R. A. Vicencio, and Yu. S. Kivshar, "Discrete solitons and nonlinear surface modes in semi-infinite waveguide arrays," Opt. Lett. 31, 1693-1695 (2006). 